Research Article

\title{
Forecasting Stock Market Volatility: A Combination Approach
}

\author{
Zhifeng Dai $\mathbb{D}$, Huiting Zhou, Xiaodi Dong, and Jie Kang \\ College of Mathematics and Statistics, Changsha University of Science and Technology, Changsha, Hunan, 410114, China \\ Correspondence should be addressed to Zhifeng Dai; zhifengdai823@163.com
}

Received 26 February 2020; Revised 12 May 2020; Accepted 18 May 2020; Published 5 June 2020

Academic Editor: Fabio Tramontana

Copyright $\odot 2020$ Zhifeng Dai et al. This is an open access article distributed under the Creative Commons Attribution License, which permits unrestricted use, distribution, and reproduction in any medium, provided the original work is properly cited.

We find that combining two important predictors, stock market implied volatility and oil volatility, can improve the predictability of stock return volatility. We also document that the stock market implied volatility provides far more significant predictability than the oil volatility and other nonoil macroeconomic and financial variables. The empirical results show the "kitchen sink" combination approach that using two predictors jointly performs better than not only the univariate regression models which use oil volatility or stock market implied volatility separately but also convex combination of the individual forecasts. This improvement of predictability is also remarkable when we consider the business cycle. Furthermore, the robust test based on different lag lengths and different macroinformation shows that our forecasting strategy is efficient.

\section{Introduction}

Prediction of stock market volatility has many important applications in risk management, asset pricing, market timing decisions, and portfolio selection. Therefore, modeling and forecasting stock market volatility is an important task and a popular research topic in financial markets [1].

The seminal paper on the economic drivers of stock market volatility is by Officer [2] which is followed by Schwert [3]. Officer [2] points towards countercyclical movements of stock market volatility, but the link between volatility and economic activity is not very strong from a statistical perspective. Christiansen et al. [4] provide a comprehensive analysis of volatility predictability in financial markets by economic variables and find that information on economic variables helps in predicting future volatility. Paye [5] shows that some variables in theory (such as national debt spreads and default gains) will affect the volatility of stocks and finds some variables that can predict stock volatility. Engle et al. [6] analyze the effect of inflation and industrial production growth on daily stock return volatility, considering each macroeconomic variable separately. Conrad and Loch [7] investigate the relationship between long-term US stock market risks and the macroeconomic environment using a two-component GARCH-
MIDAS model and show that macroeconomic variables are important determinants of the secular component of stock market volatility. In the recent years, forecasting of stock market volatility has been investigated from different perspectives, for example, Bayesian model averaging (Nonejad [8]), heterogeneous autoregressive models of realized volatility (HAR-RV) [9, 10], simple linear regression [11, 12], and mixed-frequency approach [13].

Although the prediction of stock market volatility has made good progress, there is still room for improvement. Therefore, our main goal is to obtain superior out-of-sample performance for stock market volatility prediction. In detail, firstly, we use the linear regression models which take oil volatility or stock market implied volatility as the predictor separately. Then, we utilize a "kitchen sink" combination approach that uses oil volatility and stock market implied volatility jointly.

One motivation for this paper is mainly that stock market implied volatility indices derived from option prices which reflect market's expectation on the future volatility over the remaining life of the options are generally considered to be a better measure of market's uncertainty. It is well known that the implied volatility index has long been seen as a predictor of volatility, where the GARCH model is used from an in-sample perspective (see [14-21]). In 
addition, crude oil is a core input in the modern industry. Crude oil is also one of the most important commodities. Investors' asset reallocations between commodity indices and stocks result in volatility spillovers between crude oil and stock markets (see [22-25]). It is because that stock implied volatility VIX not only contains the historical volatility information but also includes investors' expectation on the future market conditions. Oil price shocks can certainly lead to changes in stock prices by affecting real economic activities (see Kilian [26]). Therefore, we can expect that oil volatility and stock market implied volatility VIX are predictors of stock market volatility.

The other motivation for our paper is that constructing or finding new powerful predictors for stock market volatility is difficult. Therefore, we try to find existing predictors that can offer more efficient information on forecast stock volatilities and then add them into the forecast combination model to generate stock volatility forecasts. This idea is not really new because it has been well demonstrated that combination forecasts can perform even better than individual forecasts (see [27-29]). Furthermore, the forecast combination method has recently received increasing attention in the economic forecasting literature, especially in the stock return (see, for example, [30-35]). Although predictive combination is becoming more popular in stock returns, the predictability of stock return volatility is rarely used. In view of this, we consider the combined approach as an important competitive model in this paper.

We use daily data of the S\&P 500 index, the West Texas Intermediate (WTI), and the Brent crude oil price where the time is from January 1990 to December 2018 and the monthly implied volatility VIX from January 1990 to December 2018. The sum of the monthly average daily returns is built to achieve monthly volatility. The in-sample results show that there is a very significant predictability from stock market implied volatility VIX to stock market volatility.

We also take the out-of-sample $R_{\text {oos }}^{2}$ to evaluate the forecasting of out-of-sample performance as in the literature $([4,5,36,37])$. We utilize the rolling estimation and recursive estimation to produce a one-step-ahead prediction of stock market volatility from Jan. 1998 to Dec. 2018. Being of our interest, the forecasting performance of the "kitchen sink" combining the predictive regression model of oil volatility and VIX is stronger than the univariate of VIX, and it beats the univariate of oil volatility significantly during various sample periods.

The economic cyclical periods may have an impact on market predictability. To test the robustness of the predictability, we try to link market predictability to the business cyclical periods. During recession periods, $R_{\text {oos }}^{2}$ values for VIX are larger than $R_{\text {oos }}^{2}$ values for the oil volatility. The "kitchen sink" model for combining oil volatility WTI and VIX yields higher $R_{\text {oos }}^{2}$ than the regression model of VIX individually. During expansion periods, $R_{\text {oos }}^{2}$ values for all of the regressions are smaller than that during the recession periods. In addition, from a comparative perspective, the out-of-sample performance for most of the regressions is basically consistent with that during the recession periods.
To explore the predictability of our models, we use different lag lengths of stock market return realized volatility and different macroinformation to carry out the robustness analysis. Out-of-sample results show that different lag orders have little effect on out-of-sample forecasting performance for stock return volatility. In addition, the out-of-sample performance of the "kitchen sink" model by combining macroeconomic and financial variables and VIX is stronger than the univariate of macroeconomic and financial variables or VIX. Hence, our out-of-sample forecasting results over different lag lengths and different macroinformation are found to be robust.

The remainder of this paper is organized as follows: Section 2 provides our research data and summary statistics. Section 3 presents the predictive regression methodology. We report the in-sample and out-of-sample results in Section 4 and Section 5, respectively. Section 6 gives the extension analysis by linking to the business cycle. Section 7 investigates the robustness test by containing different lag lengths and different macroinformation. Finally, we conclude the paper.

\section{Data and Descriptive Statistics}

In this paper, we try to use stock implied volatility together with crude oil volatility to accurately predict the volatility of the S\&P 500 index. We utilize the daily data for the S\&P 500 index, spanning from January 1990 to December 2018. Simultaneously, we use the end-of-month closing price spanning from January 1990 to December 2018 from the implied volatility indices VIX. These data are extracted from the Thomson Reuters Database (https//www. thomsonreuters.com/en.html). In addition, we also utilize the prices of Brent crude oil and West Texas Intermediate (WTI) crude oil, which can be freely downloaded from the Energy Information Administration website [1].

Following the literature (e.g., $[4,5,12,13])$, the volatility of S\&P 500 for a month $t$ can be defined as follows:

$$
R V_{t}=\sum_{j=1}^{m} r_{t}^{2}, j, \quad j=1,2, \ldots m,
$$

where $m$ stands for the number of trading days per month and $r_{t, j}$ stands for the $j$-th daily return in the $t$-th month. The summary statistics of the basic statistics of the crude oil volatility and the volatility of S\&P 500 are reported in Table 1.

Following the literature (e.g., $[4,5,12])$, we also take the natural $\operatorname{logarithm}$ as $V_{t}=\log \left(R V_{t}\right)$, to reduce the impact of leptokurtic on the realized volatility in (1).

\section{Methodology}

3.1. Forecasting Models. For stock volatility forecasting, a standard benchmark is the following autoregressive model (AR):

$$
V_{t+1}=\omega+\sum_{i=0}^{p-1} \alpha_{i} V_{t-i}+\varepsilon_{i+1}
$$


TABLe 1: Summary statistics.

\begin{tabular}{lccccccc}
\hline & Mean & Median & S. E. & Max. & Min. & Skew. & Kurt. \\
\hline S\&P & 0.003 & 0.011 & 0.013 & 19.876 & 0.001 & 0.008 & 0.008 \\
BRT & 18.235 & 0.005 & 0.017 & 0.020 & 7.555 & 0.0001 & 0.0007 \\
WTI & 0.001 & 10.420 & 0.057 & 0.237 & 0.255 & 59.890 & 6.899 \\
VIX & 8.218 & 7.108 & 1.682 & 66.802 & 97.190 & 75.067 & 7.374 \\
\hline
\end{tabular}

Note. This table reports the basic statistics of the major regression variables, which is shown in the first column of the table. S\&P, BRT, and WTI denote the realized volatility of the S\&P500 stock index, Brent oil price, and WTI oil price, respectively. VIX denotes the implied volatility for the S\&P500 stock index. The mean value, standard deviation, median, coefficients of kurtosis and skewness, range, and the number of observations are shown in this table.

where $V_{t}=\log \left(R V_{t}\right)$ and the error term $\varepsilon_{t+1} \sim N(0,1)$, and it is assumed to be independent and identical.

Wang et al. [12] extended the benchmark AR in (2) including the volatility of oil as an additional predictor:

$$
V_{t+1}=\omega+\sum_{i=0}^{p-1} \alpha_{i} V_{t-i}+\beta V_{t, \mathrm{oil}}+\varepsilon_{i+1},
$$

where $V_{t, \text { oil }}$ is the log-realized oil volatility in the $t$-th month and the lag order $p=6$.

To investigate the predictive ability of the stock implied volatility indices VIX, we also extend the benchmark AR in (2) including a log stock market implied volatility as an additional predictor:

$$
V_{t+1}=\omega+\sum_{i=0}^{p-1} \alpha_{i} V_{t-i}+\lambda I V_{t}+\varepsilon_{i+1}
$$

where $I V_{t}$ is the stock implied volatility of the $t$-th month and $\lambda$ reflects the effect of implied volatility VIX of the $t$-th month on the $(t+1)$-th month volatility.

We will utilize the information of each forecast through the following combination of prediction methods. As in [2], we also use a "kitchen sink" model that includes stock implied volatility together with crude oil volatility in a multiple linear regression model:

$$
V_{t+1}=\omega+\sum_{i=0}^{p-1} \alpha_{i} V_{t-i}+\beta V_{t, \mathrm{oil}}+\lambda I V_{t}+\varepsilon_{i+1},
$$

where $I V_{t}$ and $V_{t, \text { oil }}$ are the stock implied volatility and the volatility of oil in the $t$-th month, respectively. In this paper, the lag order $p$ is also set to be 6 .

Another practical solution is to use a convex combination forecast method (e.g., [28]). First, individual forecasts are obtained in running predictive regressions on each predictor. Then, we can take a convex combination of the individual forecasts as the forecast:

$$
\widehat{V}_{t+1}^{\mathrm{MC}}=\lambda \widehat{V}_{t+1}^{\mathrm{oil}}+(1-\lambda) \widehat{V}_{t+1}^{I V},
$$

where $0<\lambda<1$ is the combining weights and $\widehat{V}_{t+1}^{\text {oil }}$ and $\widehat{V}_{t+1}^{I V}$ are obtained in the oil volatility-based predictive regression model (3) and stock implied volatility-based predictive model (4) in the $t$-th month, respectively. In this paper, we set $\lambda=0.1$.
3.2. Out-Of-Sample Forecast and Evaluation. For out-ofsample forecast, we use the recursive estimation method and the rolling estimation method to generate a one-step-ahead forecasting volatility. For both recursive and rolling estimation methods, the whole $T$-observation sample of oil volatility, VIX, and stock market volatility series are divided into two parts, the first $M$ observations are used for the insample and the remaining $T$ - $M$ observations are used for the out-of-sample.

Following the literature [38-43], we take a wide spread out-of-sample $R^{2}$ statistics ( $R_{\text {OoS }}^{2}$ hereafter) to evaluate the prediction performance of a given model. This statistic tests whether the out-of-sample prediction performance of the given model outperforms the benchmark. The $R_{\text {Oos }}^{2}$ is computed as

$$
R_{\mathrm{OoS}}^{2}=1-\frac{\mathrm{MSPE}_{\text {model }}}{\mathrm{MSPE}_{\text {bench }}},
$$

where $\quad \mathrm{MSPE}_{\text {model }}=(1 / T-M) \sum_{t=M+1}^{T}\left(V_{t}-\widehat{V}_{t}\right)^{2} \quad$ and $\operatorname{MSPE}_{\text {bench }}=(1 / T-M) \sum_{t=M+1}^{T}\left(V_{t}-\bar{V}_{t}\right)^{2}$. MSPE $E_{\text {bench }}$ and $\mathrm{MSPE}_{\text {model }}$ are the mean-squared predictive errors (MSPE) of the benchmark model and the tested model, respectively. In addition, the $p$ value of the one-sided test can be easily obtained from the standard normal distribution.

As by Christiansen et al. [4], Paye [5], Conrad and Loch [7], Nonejad [8], and Wang et al. [12], we also take the autoregressive model AR (6) in (2) as the benchmark model because stock realized volatility is highly persistent and the autoregressive model AR (6) is a strong benchmark for stock volatility prediction.

\section{In-Sample Analysis}

Inoue and Kilian [44] showed that it is unreasonable to obtain out-of-sample predictability without good performance for in-sample predictability. And, Table 2 reports the coefficient estimates of predictive regression models in (3), (4), and (5) which are estimated by using the ordinary least squares and $t$-statistics based on the C-W statistic method [45] which is used to test the null hypothesis of no predictability. We also give increase percentage terms in $R^{2}$ for the predictive regression models in (3), (4), and (5), relative to the benchmarks of AR (6) models in Table 2 .

The coefficient estimates of $\alpha_{1}$ and $\alpha_{2}$ in the predictive regression models in (3) and the coefficient estimate of $\alpha_{1}$ in the predictive regression models in (4) and (5) are significant and positive. It is obvious that there is volatility persistence for the stylized fact. In the regression models in (3), the estimated coefficient of $\beta$ is 0.073 and 0.112 for Brent and WTI oil volatility, respectively. And, the coefficient estimate of $\beta$ in the predictive regression models in (3) is significantly positive with $5 \%$ level for WTI oil volatility and at $10 \%$ level for Brent oil volatility. The coefficient estimate of $\lambda$ is 1.647 in the predictive regression models in (4). And, the coefficient estimate of $\lambda$ in the predictive regression models in (4) is significantly positive at $1 \%$ level for stock market implied volatility VIX, which indicates that there is a significant in-sample predictability from stock market implied volatility to stock volatility. 
TABLE 2: In-sample estimation results.

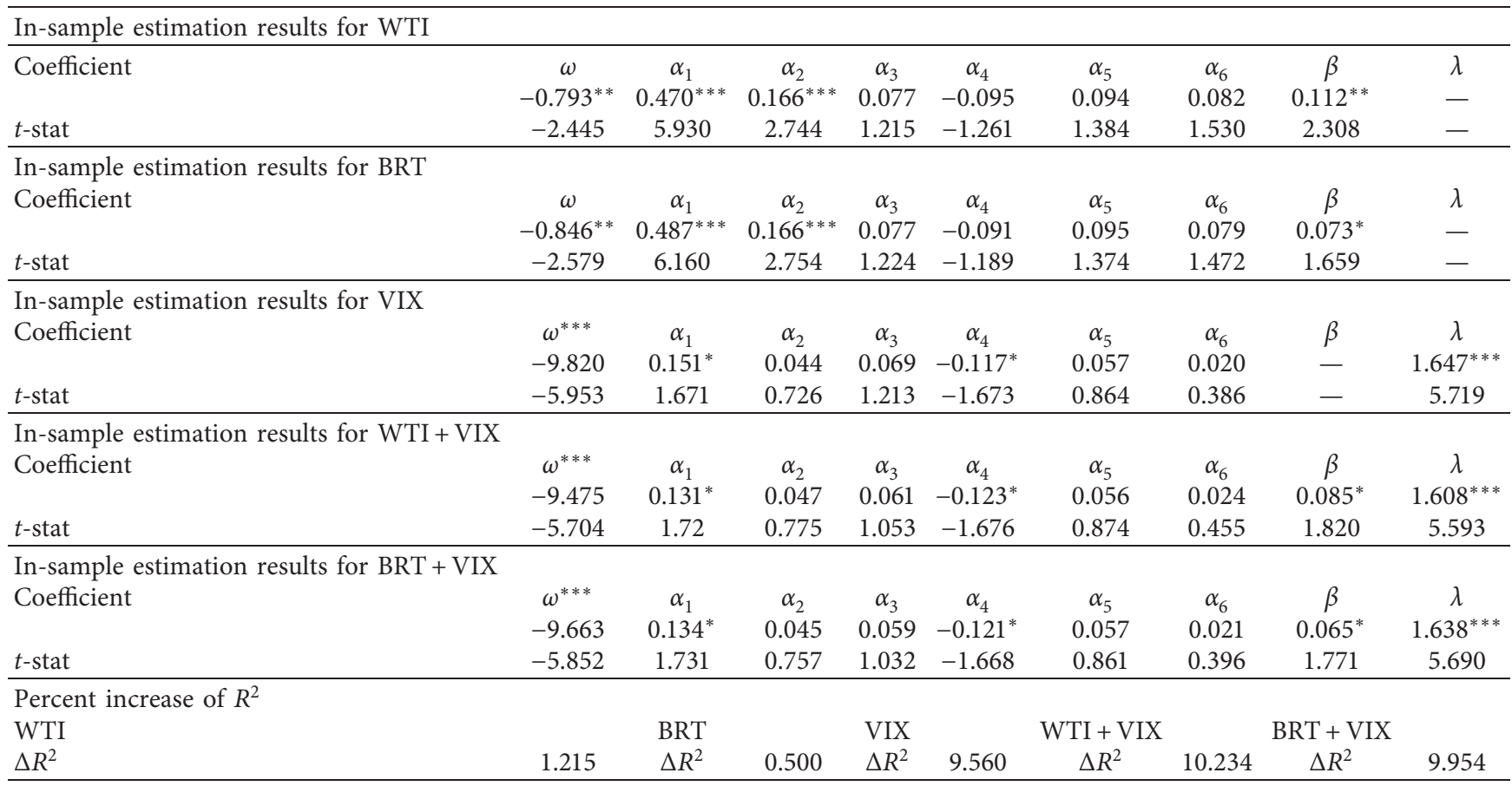

Note. This table reports the in-sample estimation results for the predictive regression models in (3), (4), and (5) for monthly stock volatility. We report the estimate of the slope coefficients, as well as the corresponding heteroskedasticity-adjusted $t$-statistic, based on the Newey-West method. We also show the percent increase in $R^{2}$ of the model of interest relative to that of the benchmark of AR in (2), The asterisks *, **, and ${ }^{* * *}$ denote rejections of null hypothesis at $10 \%, 5 \%$, and $1 \%$ significance levels, respectively.

Being of our interest, in the predictive regression models in (5), for combining WTI oil volatility and VIX, the coefficient estimate of $\beta$ is 0.085 and $\lambda$ is 1.608 for WTI oil volatility and VIX, respectively. And, the coefficient estimate of $\beta$ in the predictive regression models in (5) is significantly positive at $10 \%$ level for WTI oil volatility, and the coefficient estimate of $\beta$ in the predictive regression models in (5) is significantly positive at $1 \%$ level for VIX.

In the predictive regression models in (5), for combining Brent oil volatility and VIX, the coefficient estimate of $\beta$ is 0.065 and $\lambda$ is 1.638 for Brent oil volatility and VIX, respectively. And, the coefficient estimate of $\beta$ in the predictive regression models in (5) is significantly positive at $10 \%$ level for Brent oil volatility, and the coefficient estimate of $\beta$ in the predictive regression models in (5) is significantly positive at $1 \%$ level for VIX.

Comparing the percentage increase in $R^{2}$ of the model of interest relative to the AR benchmark (2), we can see that the values of the predictive regression models in (5) for combining oil volatility and VIX are much greater than the percentage increase in $R^{2}$ due to predictive regressions models in (3) or (4), which imply that combining oil volatility and VIX can provide more accurate prediction than most popular predictors for stock volatility.

\section{Out-Of-Sample Analysis}

Table 3 reports the predictive results for the recursive window method, where the values of $R_{\text {oos }}^{2}$ and the corresponding $p$ values of C-W statistics [45] are listed.
Firstly, we can see the predictive performance of Brent and WTI oil volatility, where the predictive regression model is used in (3). The values of $R_{\text {oos }}^{2}$ suggest that including WTI oil volatility in the regression model can result in a $1.01 \%$ decrease in MSPE during the whole out-of-sample period. The $R_{\text {oos }}^{2}$ value is larger in more recent subperiods. It is obvious that the forecasting accuracy of Brent oil volatility is a little weaker in comparison with WTI oil volatility.

The out-of-sample performance of (4) with stock market implied volatility VIX is stronger than oil volatility. The values of $R_{\text {oos }}^{2}$ suggest that including VIX in the forecasting model can cause the reduction of MSPE by $12.98 \%$ during the whole out-of-sample period. The $R_{\text {oos }}^{2}$ values are stable during the more recent subperiods. The $p$ values based on C-W statistics also show that there exists significant improvement during different periods, which indicates that the predictive ability of VIX is efficient.

Being of our interest, the out-of-sample performance of (5) for the "kitchen sink" combining oil volatility and VIX is stronger than the univariate of oil volatility or VIX. The evidence is that the values of $R_{\text {oos }}^{2}$ suggest that including VIX and oil volatility in predictive regression can result in a larger improvement of forecasting accuracy during different outof-sample periods.

The forecasting performance of the convex combination predictive model (6) for combining oil volatility and VIX is a bit weaker in comparison with the "kitchen sink" combining predictive regression model (5). The evidence is that the values of $R_{\text {oos }}^{2}$ in the convex predictive model (6) is smaller than the "kitchen sink" model (5), even smaller than VIX 
TABLE 3: Out-of-sample forecasting results, recursive window.

\begin{tabular}{|c|c|c|c|c|c|c|c|}
\hline & WTI & BRT & VIX & WTI + VIX & $\mathrm{BRT}+\mathrm{VIX}$ & $\mathrm{CC}(W+V)$ & $\mathrm{CC}(B+V)$ \\
\hline \multicolumn{8}{|c|}{ Out-of-sample forecasting results for 1998-2018 } \\
\hline$R_{\mathrm{oos}}^{2}$ & 1.01 & 0.12 & $12.98^{* * *}$ & $13.29^{* * *}$ & $13.01^{* * *}$ & $12.68^{* * *}$ & $12.58^{* * *}$ \\
\hline$p$ value & 0.1323 & 0.1963 & 0.0001 & 0.0001 & 0.0001 & 0.0001 & 0.0001 \\
\hline \multicolumn{8}{|c|}{ Out-of-sample forecasting results for 2003-2018 } \\
\hline$R_{\mathrm{oos}}^{2}$ & $1.53^{*}$ & $1.44^{*}$ & $10.41^{* * *}$ & $11.21^{* * *}$ & $10.73^{* * *}$ & $10.38^{* * *}$ & $10.24^{* * *}$ \\
\hline$p$ value & 0.0574 & 0.0654 & 0.0003 & 0.0001 & 0.0002 & 0.0003 & 0.0003 \\
\hline \multicolumn{8}{|c|}{ Out-of-sample forecasting results for 2008-2018 } \\
\hline$R_{\mathrm{oos}}^{2}$ & $2.20^{*}$ & $0.63^{*}$ & $12.32^{* * *}$ & $13.46^{* * *}$ & 12.72 & $12.08^{* * *}$ & $11.92^{* * *}$ \\
\hline$p$ value & 0.0522 & 0.0932 & 0.0014 & 0.0007 & 0.0012 & 0.0013 & 0.0014 \\
\hline \multicolumn{8}{|c|}{ Out-of-sample forecasting results for $2013-2018$} \\
\hline$R_{\text {oos }}^{2}$ & $3.43^{*}$ & 1.32 & $18.73^{* * *}$ & $20.34^{* * *}$ & $19.69^{* * *}$ & $18.08^{* * *}$ & $17.90^{* * *}$ \\
\hline$p$ value & 0.0844 & 0.1243 & 0.0060 & 0.0037 & 0.0048 & 0.0058 & 0.0061 \\
\hline
\end{tabular}

Note. This table reports the forecasting results for the predictive regression models in (3), (4), and (5) for monthly stock volatility. The table reports the out-ofsample $R^{2}$, defined in the percent reduction of the mean-squared predictive error (MSPE) of the interest models relative to that of the benchmark of AR (6). The $p$ values of Clark and West [45] (CW) tests for the equivalence of MSPEs between the interest models and the benchmark model are given in the parentheses. The asterisks ${ }^{*},{ }^{* *}$, and ${ }^{* * *}$ indicate rejections of null hypothesis at $10 \%, 5 \%$, and $1 \%$ significance levels, respectively.

over different sample periods. The reason is that the forecasting ability of oil volatility is far weaker than VIX.

Table 4 reports the predictive results for the rolling window method. Overall, the predictive performances based on the rolling window are very similar with the recursive window. From the values of $R_{\text {oos }}^{2}$, we can know that the forecasting ability of oil volatility is far weaker than VIX. In addition, the predictive performance of the regression model (5) for the "kitchen sink" combining oil volatility and VIX is stronger than the univariate of oil volatility or VIX. The forecasting performance of the convex combination predictive model (6) for combining oil volatility and VIX is a bit weaker in comparison with the "kitchen sink" combining predictive regression model (5). During different subperiods, both the $R_{\text {oos }}^{2}$ values and $p$ values of $C-W$ test [45] suggest that there is a very significant improvement of forecasting accuracy for VIX and the "kitchen sink" combining oil volatility and VIX.

\section{Business Cycles}

The economic cyclical period may have an impact on market predictability. Neely et al. [46] showed that the predictability of the stock market appears with some different results during commercial expansion and recession.

To test the predictability of crude oil volatility together with stock implied volatility, we use a business cycle indicator with NBER-date, which is equal to 1 when the economy is in recession. Further, to study the source of predictive ability, we will calculate the out-of-sample $R$ square $R_{\text {oos }}^{2}$ during business expansion and recessions periods [47-49], as follows:

$$
\begin{aligned}
& R_{\mathrm{oos}}^{2}=1-\frac{\sum_{k=1}^{q} I_{m+k}^{c}\left(V_{m+k}-\widehat{V}_{m+k}\right)^{2}}{\sum_{k=1}^{q} I_{m+k}^{c}\left(V_{m+k}-\bar{V}_{m+k}\right)^{2}}, \\
& c=\text { REC, EXP, }
\end{aligned}
$$

where $I_{m+k}^{\mathrm{REC}}\left(I_{m+k}^{\mathrm{EXP}}\right)$ is an indicator variable that equals 1 if the month $m+k$ is during a period of recession (expansion). We will give the performance of out-of-sample forecasting for different business cycles in Table 5 .

During recession periods, $R_{\text {oos }}^{2}$ values for the stock market implied volatility VIX are larger than $R_{\text {oos }}^{2}$ values for the oil volatility. The "kitchen sink" model for combining oil volatility WTI and VIX yields higher $R_{\text {oos }}^{2}$ than the regression model of VIX individually. But, the "kitchen sink" model for combining Brent oil volatility and VIX yields almost the same $R_{\mathrm{oos}}^{2}$ as the regression model of VIX individually. The forecasting performance of the convex combination predictive model (6) for combining oil volatility and VIX is a bit weaker in comparison with the "kitchen sink" combining predictive regression model (5), even for the regression model of VIX individually. During expansion periods, $R_{\text {oos }}^{2}$ values for all of the regressions are smaller than that during recession periods. In addition, from a comparative perspective, the out-of-sample forecasting performance for most of the regressions is basically consistent with that during the recession periods. Not surprisingly, the "kitchen sink" model for combining oil volatility and VIX yields higher $R_{\text {oos }}^{2}$ than the other regression models. The results in Table 5 also show that the out-of-sample results in the previous section are robust to alternative business cycles.

\section{Robustness Tests}

In this section, we will give two robustness analysis that test the predictability of our models, including different lag lengths of stock market return realized volatility and different macroinformation.

7.1. Predictability for Different Lag Lengths. For stock volatility forecasting, it is obvious that different lag orders will have impact on the prediction ability. Hence, we will consider the alternative lag orders: $2,4,6$, and 8 with the recursive window method where the out-of-sample period is 2003: 01-2018: 12 .

Table 6 reports the out-of-sample predictive results for the recursive window method with the lag orders: $2,4,6$, and 
TABLE 4: Out-of-sample forecasting results, rolling window.

\begin{tabular}{|c|c|c|c|c|c|c|c|}
\hline & WTI & BRT & VIX & WTI + VIX & $\mathrm{BRT}+\mathrm{VIX}$ & $\mathrm{CC}(W+V)$ & $\mathrm{CC}(B+V)$ \\
\hline \multicolumn{8}{|c|}{ Out-of-sample forecasting results for $1998-2018$} \\
\hline$R_{\mathrm{oos}}^{2}$ & $0.56^{*}$ & 0.17 & $12.81^{* * *}$ & $12.83^{* * *}$ & $13.70^{* * *}$ & $13.12^{* * *}$ & $13.15^{* * *}$ \\
\hline$p$ value & 0.082 & 0.117 & 0.0005 & 0.0001 & 0.0001 & 0.0001 & 0.0001 \\
\hline \multicolumn{8}{|c|}{ Out-of-sample forecasting results for 2003-2018 } \\
\hline$R_{\text {oos }}^{2}$ & $1.53^{*}$ & $1.43^{*}$ & $12.01^{* * *}$ & $14.47^{* * *}$ & $13.22^{* * *}$ & $12.66^{* * *}$ & $12.72^{* * *}$ \\
\hline$p$ value & 0.057 & 0.065 & 0.0001 & 0.0001 & 0.0001 & 0.0001 & 0.0001 \\
\hline \multicolumn{8}{|c|}{ Out-of-sample forecasting results for 2008-2018 } \\
\hline$R_{\mathrm{oos}}^{2}$ & $2.52^{*}$ & 1.43 & $12.10^{* * *}$ & $13.67^{* * *}$ & $14.38^{* * *}$ & $12.89^{* * *}$ & $12.88^{* * *}$ \\
\hline$p$ value & 0.064 & 0.113 & 0.0009 & 0.0001 & 0.0001 & 0.0006 & 0.0007 \\
\hline \multicolumn{8}{|c|}{ Out-of-sample forecasting results for 2013-2018 } \\
\hline$R_{\text {oos }}^{2}$ & $5.22^{*}$ & $4.32^{*}$ & $21.27^{* * *}$ & $22.61^{* * *}$ & $25.50^{* * *}$ & $21.67^{* * *}$ & $22.07^{* * *}$ \\
\hline$p$ value & 0.067 & 0.066 & 0.0026 & 0.0007 & 0.0002 & 0.0020 & 0.0019 \\
\hline
\end{tabular}

Note. The forecasting results for the predictive regression models in (3), (4), and (5) for monthly stock volatility. The table reports the out-of-sample $R^{2}$, defined in the percent reduction of mean-squared predictive error (MSPE) of the interest models relative to that of the benchmark of AR (6). The $p$ values of Clark and West [45] (CW) tests for the equivalence of MSPEs between the interest models and the benchmark model are given in the parentheses. The asterisks ${ }^{*}, * *$, and ${ }^{* * *}$ indicate rejections of null hypothesis at $10 \%, 5 \%$, and $1 \%$ significance levels, respectively.

TABLE 5: Out-of-sample forecasting performance over business cycles.

\begin{tabular}{|c|c|c|c|c|c|c|c|}
\hline & WTI & BRT & VIX & WTI + VIX & $\mathrm{BRT}+\mathrm{VIX}$ & $\mathrm{CC}(W+V)$ & CC $(B+V)$ \\
\hline \multicolumn{8}{|c|}{ Out-of-sample forecasting results for $1998-2018$} \\
\hline Recession period & 2.648 & 0.596 & 20.781 & 21.056 & 21.208 & 19.854 & 19.883 \\
\hline Expansion period & 0.825 & 0.116 & 12.124 & 13.553 & 13.112 & 11.896 & 11.783 \\
\hline \multicolumn{8}{|c|}{ Out-of-sample forecasting results for $2003-2018$} \\
\hline Recession period & 0.597 & 0.572 & 26.792 & 26.786 & 25.937 & 24.809 & 24.695 \\
\hline Expansion period & 1.572 & 0.191 & 8.778 & 9.659 & 9.215 & 8.945 & 8.803 \\
\hline \multicolumn{8}{|c|}{ Out-of-sample forecasting results for $2008-2018$} \\
\hline Recession period & 3.151 & 2.071 & 12.409 & 13.352 & 12.711 & 12.806 & 12.468 \\
\hline Expansion period & 1.773 & 0.425 & 6.482 & 9.787 & 9.872 & 7.531 & 6.917 \\
\hline \multicolumn{8}{|c|}{ Out-of-sample forecasting results for 2013-2018 } \\
\hline Recession period & 5.141 & 0.725 & 32.515 & 36.125 & 35.894 & 35.003 & 33.055 \\
\hline Expansion period & 1.461 & 0.555 & 7.719 & 11.011 & 10.874 & 8.367 & 7.840 \\
\hline
\end{tabular}

Note. This table reports the out-of-sample performance from statistical perspectives over business cycles based on the recursive window. The forecasting results for the predictive regression models in (3), (4), and (5) for monthly stock volatility are shown. Statistical performance is defined as out-of-sample $R$ square $\left(R_{\text {oos }}^{2}\right)$.

TABLE 6: Out-of-sample forecasting results with different lag lengths.

\begin{tabular}{|c|c|c|c|c|c|c|c|}
\hline & WTI & BRT & VIX & WTI + VIX & BRT + VIX & $\mathrm{CC}(W+V)$ & $\mathrm{CC}(B+V)$ \\
\hline \multicolumn{8}{|c|}{ Out-of-sample forecasting results with lag length 2} \\
\hline$R_{\mathrm{oos}}^{2}$ & $1.41^{*}$ & $1.32^{*}$ & $10.25^{* * *}$ & $11.18^{* * *}$ & $10.67^{* * *}$ & $10.28^{* * *}$ & $10.18^{* * *}$ \\
\hline$p$ value & 0.0765 & 0.0821 & 0.0006 & 0.0001 & 0.0002 & 0.0003 & 0.0003 \\
\hline \multicolumn{8}{|c|}{ Out-of-sample forecasting results with lag length 4} \\
\hline$R_{\text {oos }}^{2}$ & $1.48^{*}$ & $1.41^{*}$ & $10.36^{* * *}$ & $11.20^{* * *}$ & $10.70^{* * *}$ & $10.33^{* * *}$ & $10.20^{* * *}$ \\
\hline$p$ value & 0.0574 & 0.0654 & 0.0004 & 0.0001 & 0.0002 & 0.0003 & 0.0003 \\
\hline \multicolumn{8}{|c|}{ Out-of-sample forecasting results with lag length 6} \\
\hline$R_{\mathrm{oos}}^{2}$ & $1.53^{*}$ & $1.44^{*}$ & $10.41^{* * *}$ & $11.21^{* * *}$ & $10.73^{* * *}$ & $10.38^{* * *}$ & $10.24^{* * *}$ \\
\hline$p$ value & 0.0574 & 0.0654 & 0.0003 & 0.0001 & 0.0002 & 0.0003 & 0.0003 \\
\hline \multicolumn{8}{|c|}{ Out-of-sample forecasting results with lag length 8} \\
\hline$R_{\mathrm{oos}}^{2}$ & $1.50^{*}$ & $1.40^{*}$ & $10.37^{* * *}$ & $11.17^{* * *}$ & $10.67^{* * *}$ & $10.31^{* * *}$ & $10.21^{* * *}$ \\
\hline$p$ value & 0.0786 & 0.0766 & 0.0005 & 0.0003 & 0.0003 & 0.0005 & 0.0005 \\
\hline
\end{tabular}

Note. This table reports the forecasting results for the predictive regression models for monthly stock volatility with the alternative lag orders: $2,4,6$, and 8 where the out-of-sample period is 2003:01-2018:12. The table reports the out-of-sample $R^{2}$, defined in the percent reduction of the mean-squared predictive error (MSPE) of the interest models relative to that of the benchmark of AR ( $p$ ). The $p$ values of Clark and West [45] (CW) tests for the equivalence of MSPEs between the interest models and the benchmark model are given in the parentheses. The asterisks ${ }^{*},{ }^{* *}$, and ${ }^{* * *}$ indicate rejections of null hypothesis at $10 \%$, $5 \%$, and $1 \%$ significance levels, respectively. 
TABLE 7: Out-of-sample forecasting results with different macroinformation.

\begin{tabular}{|c|c|c|c|c|c|c|}
\hline \multirow{2}{*}{ Macrovariables } & \multicolumn{2}{|c|}{$1998-2018$} & \multicolumn{2}{|c|}{$2003-2018$} & \multicolumn{2}{|c|}{$2008-2018$} \\
\hline & Individual & Individual + VIX & Individual & Individual + VIX & Individual & Individual + VIX \\
\hline $\mathrm{cp}$ & 0.12 & $13.18^{* * *}$ & 0.16 & $10.78^{* * *}$ & 0.19 & $12.88^{* * *}$ \\
\hline dfr & -0.15 & $13.06^{* * *}$ & $-0.12^{* *}$ & $10.50^{* * *}$ & $-0.20^{* *}$ & $12.68^{* * *}$ \\
\hline dfy & $0.45^{* *}$ & $13.60^{* * *}$ & $0.58^{* *}$ & $11.02^{* * *}$ & $0.67^{* *}$ & $13.10^{* * *}$ \\
\hline exret & 0.11 & $13.09^{* * *}$ & 0.18 & $10.88^{* * *}$ & 0.22 & $12.68^{* * *}$ \\
\hline ip & $0.67^{* *}$ & $13.77^{* * *}$ & $0.69^{* *}$ & $11.10^{* * *}$ & $0.75^{* *}$ & $13.17^{* * *}$ \\
\hline ipvol & $1.03^{* *}$ & $14.04^{* * *}$ & $1.12^{* *}$ & $11.65^{* * *}$ & $1.22^{* *}$ & $13.89^{* * *}$ \\
\hline npv & $0.38^{*}$ & $13.56^{* * *}$ & $0.45^{*}$ & $11.05^{* * *}$ & $0.56^{* *}$ & $12.97^{* * *}$ \\
\hline ppivol & $0.22^{*}$ & $13.24^{* * *}$ & $0.30^{*}$ & $10.88^{* * *}$ & $0.36^{*}$ & $12.86^{* * *}$ \\
\hline tms & $0.32^{*}$ & $13.35^{* * *}$ & $0.40^{* *}$ & $10.97^{* * *}$ & $0.45^{* *}$ & $12.91^{* * *}$ \\
\hline hs & $0.62^{* *}$ & $13.67^{* * *}$ & $0.66^{* *}$ & $11.14^{* * *}$ & $0.72^{* *}$ & $13.12^{* * *}$ \\
\hline mkt & $0.47^{* *}$ & $13.60^{* * *}$ & $0.50^{* *}$ & $11.05^{* * *}$ & $0.55^{* *}$ & $13.06^{* * *}$ \\
\hline VIX & $12.98^{* * *}$ & - & $10.41^{* * *}$ & - & $12.32^{* * *}$ & - \\
\hline
\end{tabular}

Note. This table reports the forecasting results for the predictive regression models in (9) and (10) for monthly stock volatility. The table reports the out-ofsample $R^{2}$, defined in the percent reduction of the mean-squared predictive error (MSPE) of the interest models relative to that of the benchmark of AR (6). The $p$ values of Clark and West [45] (CW) tests for the equivalence of MSPEs between the interest models and the benchmark model are given in the parentheses. The asterisks ${ }^{*},{ }^{* *}$, and ${ }^{* * *}$ indicate rejections of null hypothesis at $10 \%, 5 \%$, and $1 \%$ significance levels, respectively.

8. From Table 6, we can see that different lag orders have little effect on out-of-sample forecasting for stock return volatility. Overall, when the lag order is 6 , the out-of-sample forecast performance is better. Therefore, a lag order of 6 is a reasonable choice.

7.2. Predictability for Different Macroinformation. Paye [5] provided a series of macroeconomic variables which can influence stock markets in terms of stock market volatility. Is it possible that the forecast improvement which can be obtained by adding macroeconomic variables to the VIX model is sensitive? To examine this question, we also consider the following models to do robustness check.

We can extend the benchmark AR in (2) including macroeconomic variables as an additional predictor:

$$
V_{t+1}=\omega+\sum_{i=0}^{p-1} \alpha_{i} V_{t-i}+\beta V_{t, \text { mav }}+\varepsilon_{i+1},
$$

where $V_{t, \text { mav }}$ is the nonoil macroeconomic variables in the $t$ th month.

As in [2], we also use a "kitchen sink" model that includes stock implied volatility together with the nonoil macroeconomic variables in a multiple linear regression model:

$$
V_{t+1}=\omega+\sum_{i=0}^{p-1} \alpha_{i} V_{t-i}+\beta V_{t, \text { mav }}+\lambda I V_{t}+\varepsilon_{i+1},
$$

where $I V_{t}$ and $V_{t, \text { mav }}$ are the stock implied volatility and the nonoil macroeconomic variables in the $t$-th month, respectively, and the lag order $p$ is also set to be 6 .

In this section, we use some macroeconomic variables for stock market activity as suggested by Paye [5]. These popular predictor variables are the commercial paper-totreasury spread (cp), default return spread (dfr), default spread (dfy), expected return (exret), growth in industrial production (ip), industrial production volatility (ipvol), net payout (npv), inflation volatility (ppivol), and term spread (tms). In addition, some other macroeconomic and financial variables are considered to be powerful predictors of volatility, for example, the US housing starts (hs) and the market factor of Fama-French three factor models (mkt).

Table 7 reports the out-of-sample forecasting results for robustness check with nonoil macroeconomic and financial variables. Firstly, all $R_{\text {oos }}^{2}$ values of the tested macroeconomic and financial variables are positive, except for $\mathrm{dfr}$, and the $R_{\text {oos }}^{2}$ values of ipvol exceeds $1 \%$. From the values of $R_{\text {oos }}^{2}$, we can know that the forecasting ability of tested macroeconomic and financial variables is far weaker than VIX.

Being of our interest, the out-of-sample performance of (10) for the "kitchen sink" combining macroeconomic and financial variables and VIX is stronger than the univariate of macroeconomic and financial variables or VIX. The evidence is that the values of $R_{\text {oos }}^{2}$ suggest that including VIX and macroeconomic and financial variables in predictive regression can result in a larger improvement of forecasting accuracy during different out-of-sample periods. The $p$ value of the C-W test [45] suggest that there is a very significant improvement of forecasting accuracy for VIX and the "kitchen sink" combining macroeconomic and financial variables and VIX. Overall, the predictive performances are robust to nonoil macroeconomic and financial variables.

\section{Conclusions}

The goal of this paper is to propose an efficient way to improve the predictability of stock volatility where we seek to use two important predictors, oil volatility and stock market implied volatility. We establish several findings. First, the stock market implied volatility extracts significantly more useful information from the predictors than the oil volatility not only in an in-sample analysis but also in an out-of-sample analysis. Second, the "kitchen sink" combination approach that uses two predictors jointly outperforms not only the univariate regression models that use each predictor's information separately but also convex combination of the individual forecasts. Our findings 
survive other extension analysis, namely, the business cycle. Further analysis demonstrates that the "kitchen sink" combination of oil volatility and stock market implied volatility contributes to improve the predictability of stock volatility over the business cycle. Finally, we test the robustness of forecast ability of different lag lengths of stock market return realized volatility and different macroinformation. The results show that the predictability is robust to different lag lengths and different macroinformation.

Our findings have some implications for market participants. Firstly, the prediction ability of stock market implied volatility is much better than that of macroeconomic and financial variables. Secondly, the "kitchen sink" combination of stock market implied volatility and macroeconomic or financial variables can improve the out-of-sample forecasting performance. Finally, the predictive power of the "kitchen sink" combination is robust to controlling the lagged volatility.

\section{Data Availability}

The data used to support the findings of this study are available from the corresponding author upon request.

\section{Conflicts of Interest}

The authors declare that they have no conflicts of interest.

\section{Acknowledgments}

This work was supported by the National Natural Science Foundation of China grants (71771030 and 11301041) and the Scientific Research Fund of the Hunan Provincial Education Department (grant no. 19A007).

\section{References}

[1] S. J. Inun, "The usefulness of cross-sectional dispersion for forecasting aggregate stock price volatility," Journal of Empirical Finance, vol. 36, pp. 162-180, 2016.

[2] R. R. Officer, "The variability of the market factor of the New York stock exchange," The Journal of Business, vol. 46, no. 3, pp. 434-453, 1973.

[3] G. W. Schwert, "Why does stock market volatility change over time?" The Journal of Finance, vol. 44, no. 5, pp. 1115-1153, 1989.

[4] C. Christiansen, M. Schmeling, and A. Schrimpf, "A comprehensive look at financial volatility prediction by economic variables," Journal of Applied Econometrics, vol. 27, no. 6, pp. 956-977, 2012.

[5] B. S. Paye, “"Déjà vol”: predictive regressions for aggregate stock market volatility using macroeconomic variables," Journal of Financial Economics, vol. 106, no. 3, pp. 527-546, 2012.

[6] R. F. Engle, E. Ghysels, and B. Sohn, "Stock market volatility and macroeconomic fundamentals," Review of Economics and Statistics, vol. 95, no. 3, pp. 776-797, 2013.

[7] C. Conrad and K. Loch, "Anticipating long-term stock market volatility," Journal of Applied Econometrics, vol. 30, no. 7, pp. 1090-1114, 2015.

[8] N. Nonejad, "Forecasting aggregate stock market volatility using financial and macroeconomic predictors, which models forecast best, when and why," Journal of Empirical Finance, vol. 42, pp. 131-154, 2018.

[9] X. Gong and B. Lin, "Forecasting the good and bad uncertainties of crude oil prices using a HAR framework," Energy Economics, vol. 67, pp. 315-327, 2018.

[10] Y. Wang, C. Wu, and L. Yang, "Forecasting crude oil market volatility: a markov switching multifractal volatility approach," International Journal of Forecasting, vol. 32, no. 1, pp. 1-9, 2016.

[11] J. B. Feng, Y. D. Wang, and L. B. Yin, "Oil volatility risk and stock market volatility predictability, evidence from $\mathrm{g} 7$ countries," Energy Economics, vol. 68, pp. 240-254, 2018.

[12] Y. Wang, Y. Wei, C. Wu, and L. Yin, "Oil and the short-term predictability of stock return volatility," Journal of Empirical Finance, vol. 47, pp. 90-104, 2018.

[13] Y. Zhang, F. Ma, T. Wang, and L. Liu, "Out-of-sample volatility prediction, a new mixed- frequency approach," Journal of Forecasting, vol. 28, no. 7, pp. 1-23, 2019.

[14] D. P. Chiras and S. Manaster, "The information content of option prices and a test of market efficiency," Journal of Financial Economics, vol. 6, no. 2-3, pp. 213-234, 1978.

[15] B. J. Christensen and N. R. Prabhala, "The relation between implied and realised volatility," Journal of Financial Economics, vol. 50, pp. 125-150, 1998.

[16] C. J. Corrado and T. W. Miller Jr., "The forecast quality of cboe implied volatility indexes," Journal of Futures Markets, vol. 25, no. 4, pp. 339-373, 2005.

[17] P. Giot, "Implied volatility indexes and daily value at risk models," The Journal of Derivatives, vol. 12, no. 4, pp. 54-64, 2005.

[18] F. Wen, Y. Zhao, M. Zhang, and C. Hu, "Forecasting realized volatility of crude oil futures with equity market uncertainty," Applied Economics, vol. 51, no. 59, pp. 6411-6427, 2019.

[19] F. Wen, L. Xu, G. Ouyang, and G. Kou, "Retail investor attention and stock price crash risk: evidence from China," International Review of Financial Analysis, vol. 65, Article ID 101376, 2019.

[20] R. Becker, A. E. Clements, and S. I. White, "Does implied volatility provide any information beyond that captured in model-based volatility forecasts?" Journal of Banking \& Finance, vol. 31, no. 8, pp. 2535-2549, 2007.

[21] D. Kambouroudis and D. McMillan, "Does vix or volume improve garch volatility forecasts?" Applied Economics, vol. 48, pp. 1-19, 2016.

[22] M. Aktham, B. Awartani, and E. Bouri, "The directional volatility connectedness between crude oil and equity markets: new evidence from implied volatility indexes," Energy Economics, vol. 57, pp. 78-93, 2016.

[23] Q. Ji, E. Bouri, and D. Roubaud, "Dynamic network of implied volatility transmission among US equities, strategic commodities, and brics equities," International Review of Financial Analysis, vol. 57, pp. 1-12, 2018.

[24] E. Bouri, D. Lien, D. Roubaud, and S. J. H. Shahzad, "Directional predictability of implied volatility: from crude oil to developed and emerging stock markets," Finance Research Letters, vol. 27, pp. 65-79, 2018.

[25] F. Wen, N. Wu, and X. Gong, "China's carbon emissions trading and stock returns," Energy Economics, vol. 86, p. 104627, 2020.

[26] L. Kilian, "Not all oil price shocks are alike: disentangling demand and supply shocks in the crude oil market," American Economic Review, vol. 99, no. 3, pp. 1053-1069, 2009. 
[27] J. M. Bates and C. W. J. Granger, "The combination of forecasts," Journal of the Operational Research Society, vol. 20, no. 4, pp. 451-468, 1969.

[28] A. Timmermann, "Forecast combinations," in Handbook of Economic Forecasting, G. Elliott, C. W. J. Granger, and A. Timmermann, Eds., pp. 135-196, Elsevier, Amsterdam, Netherlands, 2006.

[29] D. E. Rapach, J. K. Strauss, and G. Zhou, "Out-of-sample equity premium prediction: combination forecasts and links to the real economy," Review of Financial Studies, vol. 23, no. 2, pp. 821-862, 2010.

[30] P. Poncela, J. Rodríguez, R. Sánchez-Mangas, and E. Senra, "Forecast combination through dimension reduction techniques," International Journal of Forecasting, vol. 27, no. 2, pp. 224-237, 2011.

[31] Z. Dai and F. Wen, "Some improved sparse and stable portfolio optimization problems," Finance Research Letters, vol. 27, pp. 46-52, 2018.

[32] H. Lin, C. Wu, and G. Zhou, "Forecasting corporate bond returns with a large set of predictors: an iterated combination approach," Management Science, vol. 64, no. 9, pp. 4218-4238, 2018.

[33] S. Jordan, A. Vivian, and M. Wohar, "Forecasting market returns, bagging or combining?" International Journal of Forecasting, vol. 33, no. 1, pp. 102-120, 2018.

[34] Z. Dai, X. Chen, and F. Wen, “A modified perry's conjugate gradient method-based derivative-free method for solving large-scale nonlinear monotone equations," Applied Mathematics and Computation, vol. 270, pp. 378-386, 2015.

[35] X. Zhu and J. Zhu, "Predicting stock returns: a regimeswitching combination approach and economic links," Journal of Banking \& Finance, vol. 37, no. 11, pp. 4120-4133, 2013.

[36] Z. Dai and H. Zhou, "Prediction of stock returns: sum-of-theparts method and economic constraint method," Sustainability, vol. 12, no. 2, p. 541, 2020.

[37] Z. Dai, H. Zhou, F. Wen, and S. He, "Efficient predictability of stock return volatility: the role of stock market implied volatility," The North American Journal of Economics and Finance, vol. 52, Article ID 101174, 2020.

[38] J. Y. Campbell and S. B. Thompson, "Predicting excess stock returns out of sample: can anything beat the historical average?" Review of Financial Studies, vol. 21, no. 4, pp. 15091531, 2008.

[39] Z. Dai and H. Zhu, "Forecasting stock market returns by combining sum-of-the-parts and ensemble empirical mode decomposition," Applied Economics, vol. 52, no. 21, pp. 2309-2323, 2020.

[40] J. Fleming, C. Kirby, and B. Ostdiek, "The economic value of volatility timing using "realized" volatility," Journal of Financial Economics, vol. 67, no. 3, pp. 473-509, 2003.

[41] Z. Dai and H. Zhu, "A modified hestenes-stiefel-type derivative-free method for large-scale nonlinear monotone equations," Mathematics, vol. 8, no. 2, p. 168, 2020.

[42] M. Guidolin and A. Timmermann, "Asset allocation under multivariate regime switching," Journal of Economic Dynamics and Control, vol. 31, no. 11, pp. 3503-3544, 2007.

[43] Z. Dai, X. Dong, J. Kang, and L. Hong, "Forecasting stock market returns: new technical indicators and two-step economic constraint method," The North American Journal of Economics and Finance, vol. 53, Article ID 101216, 2020.

[44] A. Inoue and L. Kilian, "In-sample or out-of-sample tests of predictability, which one should we use?" Econometric Review, vol. 23, no. 4, pp. 371-402, 2004.
[45] T. E. Clark and K. D. West, "Approximately normal tests for equal predictive accuracy in nested models," Journal of Econometrics, vol. 138, no. 1, pp. 291-311, 2007.

[46] C. J. Neely, D. E. Rapach, J. Tu, and G. Zhou, "Forecasting the equity risk premium: the role of technical indicators," Management Science, vol. 60, no. 7, pp. 1772-1791, 2014.

[47] Z. Dai and H. Zhu, "Stock return predictability from a mixed model perspective," Pacific-Basin Finance Journal, vol. 60, p. 101267, 2020.

[48] Y. Zhang, F. Ma, and Y. Wang, "Forecasting crude oil prices with a large set of predictors: can lasso select powerful predictors?" Journal of Empirical Finance, vol. 54, pp. 97-117, 2019.

[49] Z. Dai, H. Zhu, and F. Wen, "Two nonparametric approaches to mean absolute deviation portfolio selection model," Journal of Industrial and Management Optimization, 2019. 